BULETINUL INSTITUTULUI POLITEHNIC DIN IAȘI

\section{sciendo}

DOI: 10.2478/BIPMF-2021-0011
(BULLETIN OF THE POLYTECHNIC INSTITUTE FROM IAȘI)

Published by "Gheorghe Asachi" Technical University of Iasi

Volume 67(71), No. 2, 2021

Section Mathematics Theoretical Mechanics. Physics

\title{
ON THE HOLOGRAPHIC TYPE DYNAMICS IN COMPLEXITY ECONOMICS
}

\section{ȘTEFANA AGOP ${ }^{1}$, GAVRIL ȘTEFAN ${ }^{1}$, TUDOR-CRISTIAN PETRESCU ${ }^{2}$, ALEXANDRA SAVIUC ${ }^{3, *}$ and CRISTINA-MARCELA RUSU ${ }^{4}$}

\author{
${ }^{1}$ Iaşi University of Life Sciences, Department of Agroeconomy, Iaşi, Romania \\ 2"Gheorghe Asachi" Technical University of Iaşi, \\ Department of Structural Mechanics, Iași, Romania \\ 3"Alexandru Ioan Cuza" University of Iaşi, \\ Department of Physics, Iaşi, Romania \\ 4"Gheorghe Asachi” Technical University of Iaşi, \\ Department of Physics, Iaşi, Romania
}

Received: May 7, 2021

Accepted for publication: June 14, 2021

\begin{abstract}
Assimilating any complex economic system with a fractal, in the most general Mandelbrot's sense, non - differentiable behaviors in its economic dynamics are analyzed. As such, economic dynamics in the form of Schrödinger - type various regimes imply "holographic implementations" of the economic processes through group invariance of SL(2R) - type. Then, by means of previous group invariance as synchronization group between any economic system entities, both the phases and the amplitudes of the entities are affected from a homographic perspective. The usual "synchronization" manifested through the delay of the amplitudes and phases of the entities of the economic system must represent here only a fully particular case. In a special case of synchronization of economic system entities, given by Riccati type gauge, period doubling, damping oscillations, self - modulation and chaotic regimes emerge as natural behaviors in the economic dynamics of the economic processes.
\end{abstract}

*Corresponding author; e-mail: alexandra.saviuc@iroiasi.ro

(C) 2021 Agop Ș. et al. This is an Open access article licensed under the Creative Commons Attribution-Non Commercial - No Dericatives 4.0 International License (CC BY-NV-ND 4.0) 
Keywords: fractal theory of motion; group invariances of SL(2R) - type; complexity economics; complex system.

\section{Introduction}

As with many systems that are in the service of Humanity, economics is one of the oldest and, arguably, most complex ones. In fact, there exists a scientific branch called complexity economics, which studies economics and associated phenomena through the lens of complex system theory.

Complex system theory postulates that, in such a system, the individual entities interact with each other in a non-linear manner, in such a way that the outcome of such interactions is the result of "collective" actions, rather than the "individual" ones. Thus, non-linear phenomena such as self-structuring, chaoticity etc. emerge as "natural consequences" of the existence of a complex system. Furthermore, complex systems can interact with other complex systems (Arthur, 2013; Bar-Yam, 1999; Mitchell, 2011; Politi and Badii, 2003).

Taking the above considerations into account when talking about any economic system/complexity economics, the appearance of economic structures, trends, models, patterns etc. is studied, with individual optimizations and system equilibrium usually not being taken into account. In a complex economic system, events such as inflation are viewed as emergent phenomena, since they are the result of the interactions between the complex system's entities (i.e. humans, companies etc.) and its economic regulatory mechanisms (i.e. complex system feedback structures), which ultimately produce a result expected by the majority. Complexity economics theory gained significance after the 2008 financial crisis, when well-established theories had little influence in the unfolding of the associated economic events (Battiston et al., 2016).

In the present paper, the authors propose a fractal model for the study of complex economic phenomena.

\section{Mathematical Model}

The fundamental assumption of the author's proposed model is the one that the dynamics of any entity belonging to the economic system will be represented by continuous but non - differentiable motion curves (fractal motion curves). These fractal motion curves exhibit the property of selfsimilarity in its every point, which can be rewritten into a property of holography (every part reflects the whole) (Nottale, 2011; Mandelbrot, 1982). Basically, the discussion will be about "holographic implementations" of dynamics of any complex economic system, for example through Schrödingertype fractal "regimes" (i.e. describing economic system dynamics through 
Schrödinger - type equations at various scale resolutions - Schrödinger equation of fractal type) in the framework of the Fractal Theory of Motion (Agop and Mercheş, 2018; Agop and Păun, 2017).

In what follows, let it be considered that the scale covariance principle (the econophysics laws applied to economic system dynamics are invariant with respect to scale resolution transformations (Nottale, 2011)) and postulate that the evolution from the standard (differentiable) economic dynamics to the fractal (non - differentiable) economic dynamics can be achieved by changing the standard time derivative $\frac{d}{d t}$ by the non - differentiable operator $\frac{\hat{d}}{d t}$ (Agop and Mercheş, 2018; Agop and Păun, 2017)

$$
\frac{\hat{d}}{d t}=\partial_{t}+\widehat{V}^{l} \partial_{l}+\frac{1}{4}(d t)^{\left(\frac{2}{D_{F}}\right)-1} D^{l p} \partial_{l} \partial_{p}
$$

where

$$
\begin{gathered}
\hat{V}^{l}=V_{D}^{l}-V_{F}^{l} \\
D^{l p}=d^{l p}-i \bar{d}^{l p} \\
d^{l p}=\lambda_{+}^{l} \lambda_{+}^{p}-\lambda_{-}^{l} \lambda_{-}^{\underline{p}} \\
\bar{d}^{l p}=\lambda_{+}^{l} \lambda_{+}^{p}+\lambda_{-}^{l} \lambda_{-}^{p} \\
\partial_{t}=\frac{\partial}{\partial t}, \partial_{l}=\frac{\partial}{\partial X^{l}}, \partial_{l} \partial_{p}=\frac{\partial}{\partial X^{l}} \frac{\partial}{\partial X^{p}}, i=\sqrt{-1}, \quad l, p=1,2,3
\end{gathered}
$$

In the previous relations $\hat{V}^{l}$ is the complex velocity, $V_{D}^{l}$ is the differentiable velocity independent on the scale resolution $d t$ and $V_{F}^{l}$ is the non differentiable velocity dependent on the scale resolution. $X^{l}$ is the fractal spatial coordinate and $t$ is the non-fractal time having the character of an affine framework of the motion curves. $D^{l p}$ represents the constant tensor associated with the differentiable - non - differentiable transition of the economic dynamics, $\lambda_{+}^{l}$ is the constant vector associated with the leading differentiable non - differentiable of economic processes and $\lambda_{-}^{l}$ is the constant vector correlated with the backwards differentiable - non - differentiable of economic processes. $D_{F}$ is the fractal dimension of the movement curve. For the fractal dimension it is possible to adopt any definition: Kolmogorov type fractal dimension, Hausdorff - Besikovici type fractal dimension etc. (Mandelbrot, 1982; Jackson, 1991; Cristescu, 2008). But once appointed this becomes operational, it needs to be constant and arbitrary: $D_{F}<2$ for the corelative economic processes, $D_{F}>2$ for the non-corelative economic processes etc. (Nottale, 2011; Mandelbrot, 1982). 
Now, the non - differentiable operator achieves the character of the scale covariant derivative. Particularly, it is used to formulate the fundamental equations of the economic dynamics, in the same form as in the classic (differentiable) case. In this case, accepting the functionality of the scale covariant principle, i.e. applying scale covariant derivative (1) to the complex velocity field (2), in the absence of any external constraint, the geodesics equation of the economic dynamics takes the form (Agop and Mercheş, 2018; Agop and Păun, 2017; Gavriluț et al., 2019; Mercheş and Agop, 2016; Mazilu et al., 2019):

$$
\frac{\hat{d} \widehat{V}^{i}}{d t}=\partial_{t} \hat{V}^{i}+\hat{V}^{l} \partial_{l} \widehat{V}^{i}+\frac{1}{4}(d t)^{\left(\frac{2}{D_{F}}\right)-1} D^{l k} \partial_{l} \partial_{k} \widehat{V}^{i}=0
$$

This implies that the fractal local acceleration $\partial_{t} \hat{V}^{i}$, the fractal convection $\widehat{V}^{l} \partial_{l} \widehat{V}^{i}$ and the fractal dissipation $D^{l k} \partial_{l} \partial_{k} \widehat{V}^{i}$ of any economic system entity, make their balance in any point of the motion fractal curve. More that that, the presence of the complex coefficient of viscosity - type $4^{-1}(d t)^{\left(\frac{2}{D_{F}}\right)-1} D^{l k}$ in the economic system dynamics establishes that it is a "rheological" medium. So, the economic structures have memory, as a datum, by their own structure (it is known the fact that any economic crisis, for example the one of 2008, is related to past events which led to its unfolding).

In case the fractalization in the dynamics of economics is achieved by Markov - type stochastic processes, which involve Lévy type movements (Jackson, 1991; Cristescu, 2008) of the economic entities, then:

$$
\lambda_{+}^{i} \lambda_{+}^{l}=\lambda_{-}^{i} \lambda_{-}^{l}=2 \lambda \delta^{i l}
$$

Where $\lambda$ represents a coefficient related to the differentiable - non differentiable transition and $\delta^{i l}$ is Kronecker's pseudo - tensor.

Taking into consideration all of the above, the geodesics equation takes the simple form:

$$
\frac{\hat{d} \widehat{V}^{i}}{d t}=\partial_{t} \widehat{V}^{i}+\hat{V}^{l} \partial_{l} \widehat{V}^{i}-i \lambda(d t)^{\left(\frac{2}{D_{F}}\right)-1} \partial^{l} \partial_{l} \widehat{V}^{i}=0
$$

For irrotational motions of the economic entities, the complex velocity field $\hat{V}^{i}$ becomes:

$$
\widehat{V}^{i}=-2 i \lambda(d t)^{\left(\frac{2}{D_{F}}\right)-1} \partial^{i} \ln \Psi
$$

Then replacing (6) in (5), the geodesics Eq. (5) becomes Schrödinger type equation at various scale resolutions (Schrödinger equation of fractal type): 


$$
\lambda^{2}(d t)^{\left(\frac{4}{D_{F}}\right)-2} \partial^{l} \partial_{l} \Psi+i \lambda(d t)^{\left(\frac{2}{D_{F}}\right)-1} \partial_{t} \Psi=0
$$

The variable $\Phi=-2 i \lambda(d t)^{\left(2 / D_{F}\right)-1} \ln \Psi$ describes, through (6), the complex scalar potential of the complex velocity field, while $\Psi$ coincide to the state function of fractal type. Both variables, $\Phi$ and $\Psi$, have no direct economic connotation, but possible "mixes" of them can acquire it if they appease certain conservation laws.

Let it be made explicit such a situation for $\Psi$. In order for this to happes, it is first noticed that the complex conjugate of $\Psi$, that is $\bar{\Psi}$, satisfies through (7) the equation:

$$
\lambda^{2}(d t)^{\left(\frac{4}{D_{F}}\right)-2} \partial^{l} \partial_{l} \bar{\Psi}-i \lambda(d t)^{\left(\frac{2}{D_{F}}\right)-1} \partial_{t} \bar{\Psi}=0
$$

Multiplying (7) by $\bar{\Psi}$ and (8) by $\Psi$, subtracting the results and introducing the notations:

$$
\rho=\Psi \bar{\Psi}, \quad J=i \lambda(d t)^{\left(\frac{4}{D_{F}}\right)-1}(\Psi \nabla \bar{\Psi}-\bar{\Psi} \nabla \Psi)
$$

it is possible to obtain the conservation law of states density of fractal type:

$$
\partial_{t} \rho+\nabla J=0
$$

In (10) $\rho$ corresponds to the states density of fractal type and $\boldsymbol{J}$ corresponds to the states density current of fractal type.

\subsection{Economic Dynamics with Invariance Group of SL(2R) - Type by Means of Riccati Gauge}

The idea of motion equation has an enlarged significance, starting with the Fractal Theory of Motion under the form of Scale Relativity (Agop and Păun, 2017; Agop and Mercheş, 2018). Let it be noted that Schrödinger's equation of fractal type - motion equation for the state function $\Psi$ of fractal type - besides the fact that it is invariant with respect to the Galilei vectorial transformation group, it is also invariant, in a separate way, to time transformations and one dimensional coordinates (let it be $\mathrm{x}$ ) represent a group in themselves (Postnikov, 1985; Simon, 2008). They constitute, in the most general case of motion in a single direction, a realization of the group SL(2R) (Simon, 2008; Mazilu and Agop, 2012), but with two variables and three parameters, through the action (Mazilu and Agop, 2012; De Alfaro et al., 1976; Niederer, 1972; Deffner and Campbell, 2019; Gemmer et al., 2004):

$$
t^{\prime} \rightarrow \frac{\alpha t+\beta}{\gamma t+\delta}, \quad x^{\prime}=\frac{x}{\gamma t+\delta}
$$


Every vector in the tangent space $\mathrm{SL}(2 \mathrm{R})$ represents a linear combination of the three fundamental vectors, the infinitesimal action generators:

$$
X_{1}=\frac{\partial}{\partial t}, \quad X_{2}=t \frac{\partial}{\partial t}+\frac{x}{2} \frac{\partial}{\partial x}, \quad X_{3}=t^{2} \frac{\partial}{\partial t}+t x \frac{\partial}{\partial x}
$$

These appease the main structure equations:

$$
\left[X_{1}, X_{2}\right]=X_{1}, \quad\left[X_{2}, X_{3}\right]=X_{3}, \quad\left[X_{3}, X_{1}\right]=-2 X_{2}
$$

which are taken as standard commutation relations for this type of algebraic structure. The group has an invariant function, which can be achieved as the solution of a partial differential equation:

$$
\left(c X_{1}+2 b X_{2}+a X_{3}\right) f(t, x)=0
$$

which, in view of (12), means:

$$
\left(a t^{2}+2 b t+c\right) \frac{\partial f(t, x)}{\partial t}+(a t+b) x \frac{\partial f(t, x)}{\partial x}=0
$$

The general solution of this equation represents a function of the arbitrary value of the ratio:

$$
\frac{x^{2}}{a t^{2}+2 b t+c}
$$

which represent the different path of transitivity of the action described by operators from (2). In the particular case in which such a function is linear and, moreover, is a constant, it is possible to state that it represents a motion equation, be it a free entity, either a geodesic motion on the surface of a cone, or a non - standard interpretation if the statistical description is pertinent regarding the argument.

It is understood that the motion equation is linked, according to these interpretations, to the invariant functions of the $\operatorname{SL}(2 \mathrm{R})$ algebra which, as an idea, may be introduced here by a generalization of the grouping procedure.

Then, in the first of Eqs. (11), this requirement would mean that the economic system entities are considered simultaneously. Each entity can be located in the swarm by four homogenous coordinates $(\alpha, \beta, \gamma, \delta)$, or three nonhomogenous coordinates, if the Eq. (11) represents the context of time and a one - dimensional coordinate for the space domain, covered by this economic system. The simultaneity condition of the free entities of the swarm can be differently characterized, from a Riccati equation in pure differentials (i.e. through a gauge of Riccati type) (Mazilu and Agop, 2012): 


$$
d \frac{\alpha t+\beta}{\gamma t+\delta}=0, d t=\omega^{1} t^{2}+\omega^{2} t+\omega^{3}
$$

Thus, for the description of the economic dynamics as a succession of states of an ensemble of simultaneous entities, as it were, it is enough to have three differentiable 1 - forms, representing a co-frame of SL(2R) algebra (Mazilu and Agop, 2012):

$$
\begin{gathered}
\omega^{1}=\frac{\alpha d \gamma-\gamma d \alpha}{\alpha \delta-\beta \gamma} \\
\omega^{2}=\frac{\alpha d \delta-\delta d \alpha+\beta d \gamma-\gamma d \beta}{\alpha \delta-\beta \gamma} \\
\omega^{3}=\frac{\beta d \delta-\delta d \beta}{\alpha \delta-\beta \gamma}
\end{gathered}
$$

That this co-frame point out to such an algebra, can be verified by direct calculation of the Maurer - Cartan equations [Cartan], which are characteristic:

$$
\begin{gathered}
d \wedge \omega^{1}-\omega^{1} \wedge \omega^{2}=0 \\
d \omega^{2}+2\left(\omega^{3} \wedge \omega^{1}\right)=0 \\
d \omega^{3}-\omega^{2} \wedge \omega^{3}=0
\end{gathered}
$$

It was shown by Élie Cartan that under these conditions, one can prove that the Eq. (17) is an exact differential (Cartan, 1951; Cartan, 2001), consequently it should always have an integral. The Cartan - Killing metric of this co-frame is given by the quadratic form (Mazilu and Agop, 2012):

$$
d s^{2}=\frac{1}{4}\left(\omega_{2}^{2}-4 \omega_{1} \omega_{2}\right)=\frac{(\alpha d \delta+\delta d \alpha-\beta d \gamma-\gamma d \beta)^{2}}{4(\alpha \delta-\beta \gamma)^{2}}-\frac{d \alpha d \delta-d \beta d \gamma}{\alpha \delta-\beta \gamma}
$$

so that a state of an economic system in a given dynamic can be organized as a metric plan space, i.e. a Riemannian three - dimensional space (Mazilu and Agop, 2012). The geodesics of this Riemannian space are given by some conservation laws of equations:

$$
\omega^{1}=a^{1} d \tau, \quad \omega^{2}=2 a^{2} d \tau, \quad \omega^{3}=a^{3} d \tau
$$

where $a^{1}, a^{2}, a^{3}$ are constant and $\theta$ is the affine parameter of the geodesics, so that, along these geodesics of differential Eq. (17) is an ordinary differential of Riccati type: 


$$
\frac{d t}{d \tau}=a^{1} t^{2}+2 a^{2} t+a^{3}
$$

Mathematically speaking, this problem requires an ensemble generated by a harmony mapping between the positions in space and the economic system entities, with the square of the coordinate $x$ measuring the variance of the distribution which describes the spreading of economic system entities in space.

Because in economic systems the time, $t$, is inversely proportional with the "temperature", $T$ (as a measure of the structuring degree of the economic system) (Mantegna and Stanley, 2016) under the shape of $t \equiv \frac{\mu}{T}$ with $\mu=$ const., Eq. (22) with the substitutions:

$$
-\frac{\mu}{a^{3}}=A, \quad-\frac{a^{2} \mu}{a^{3}}=B, \quad-\frac{a_{1} \mu^{2}}{a^{3}}=A C
$$

takes the shape:

$$
A \frac{d T}{d \tau}-T^{2}+2 B T+A C=0
$$

Because the roots of the polynom:

$$
P(T)=T^{2}-2 B T-A C
$$

can be written in the shape:

$$
\begin{gathered}
T_{1}=B+i A \Omega \\
T_{2}=B-i A \Omega \\
\Omega^{2}=\frac{C}{A}-\left(\frac{B}{A}\right)^{2}
\end{gathered}
$$

the change of variable:

transforms the Eq. (24) in:

$$
z=\frac{T-T_{1}}{T-T_{2}}
$$

of solution:

$$
\dot{z}=z i \Omega z
$$

$$
z(\tau)=z(0) e^{z i \Omega z}
$$

As such, if the initial condition $z(0)$ is conveniently expressed, then it is possible to construct the general solution of Eq. (24), by writing the transformation (27) in the shape: 


$$
T=\frac{T_{1}+r e^{2 i \Omega\left(\tau-\tau_{0}\right)} T_{2}}{1+r e^{2 i \Omega\left(\tau-\tau_{0}\right)}}
$$

where $r$ and $\tau_{0}$ are two integration constants. Using relations (26), it is possible to write this solution in real terms:

$z=B+A \Omega\left\{\frac{2 r \sin \left[2 \Omega\left(\tau-\tau_{0}\right)\right]}{1+r^{2}+2 r \cos \left[2 \Omega\left(\tau-\tau_{0}\right)\right]}+i \frac{1-r^{2}}{1+r^{2}+2 r \cos \left[2 \Omega\left(\tau-\tau_{0}\right)\right]}\right\}$

Therefore, synchronization of phase - amplitude type between economic entities in the economic dynamics process implies group invariance of SL(2R) type. Then, period doubling, damping oscillations, self - modulation and chaotic regimes emerge as natural behaviors in the economic systems (Fig. 1 for $r=0.5$ and Real $[(z-B) / A] \equiv$ Amplitude at various scale resolutions, given by means of the maximum value of $\Omega$ ).
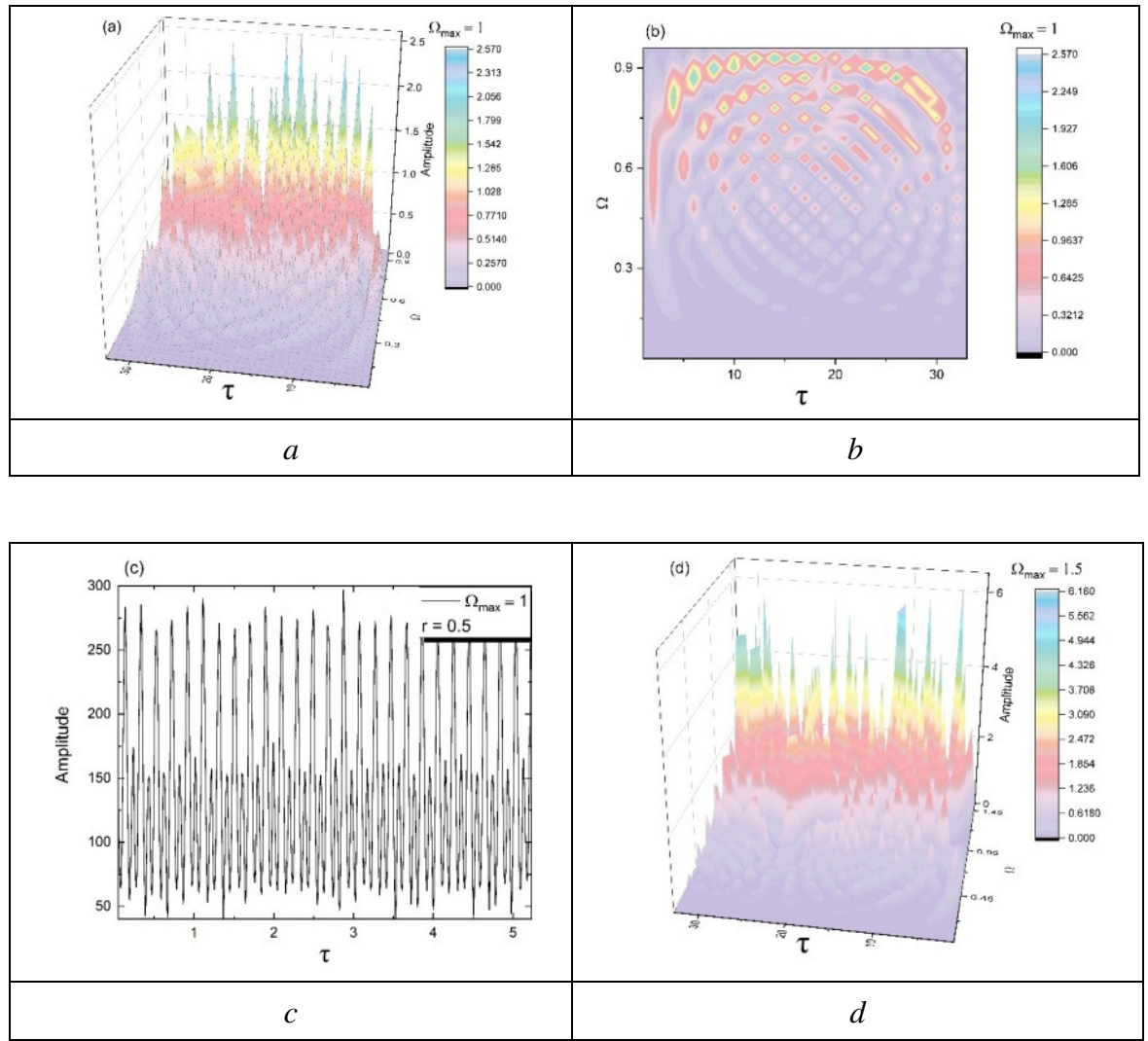

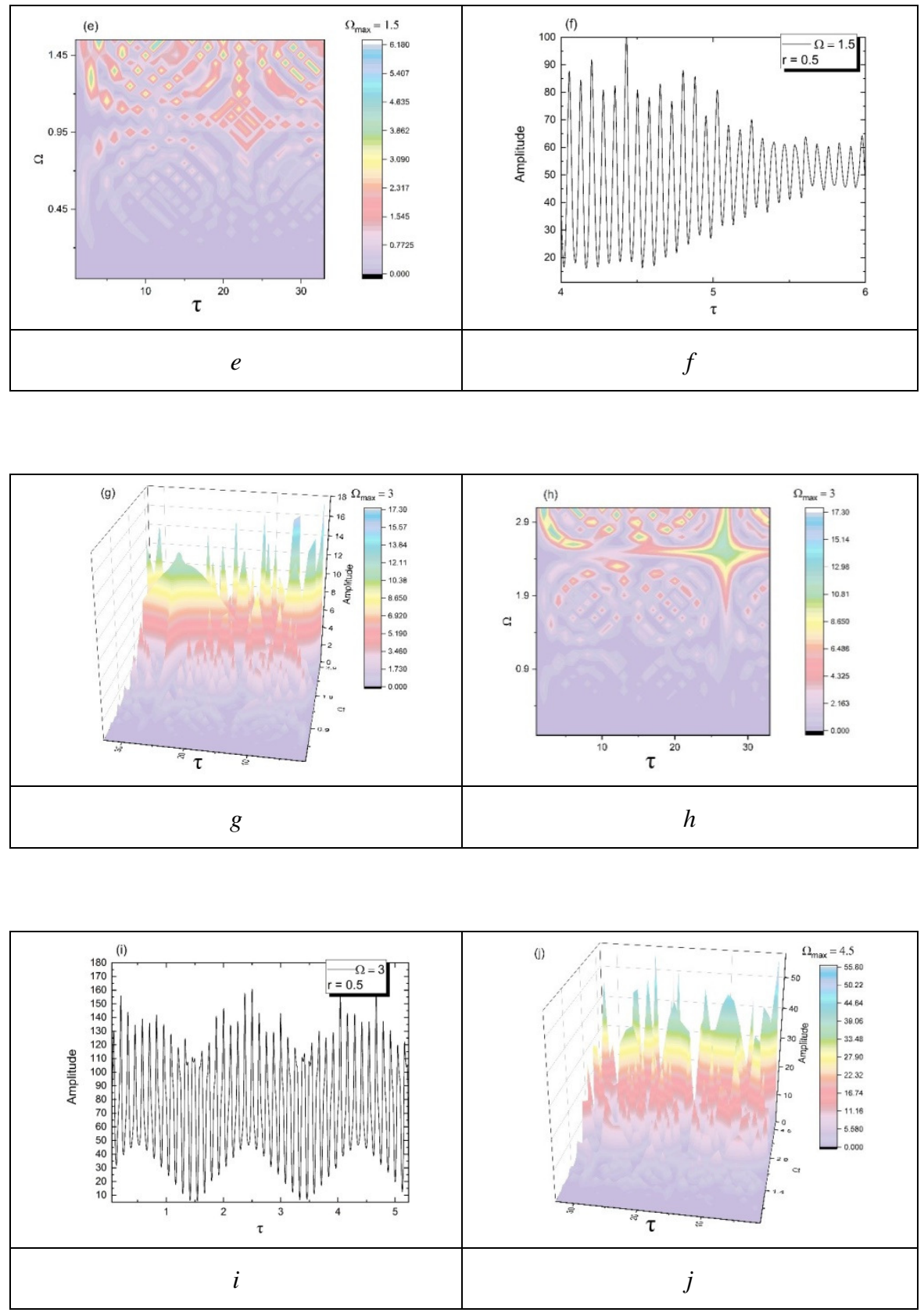


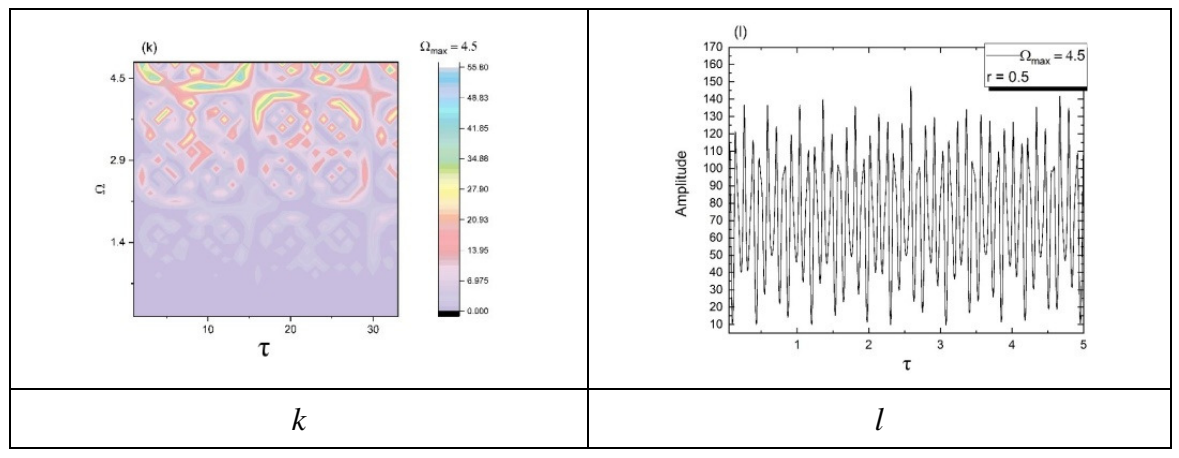

Fig. 1 - Various types of evolutions during economic processes (3D, contour plot and time series) representation: period doubling $(a, b, c)$, damped oscillation regimes $(d, e, f)$, signal modulation $(g, h, i)$ and chaotic behavior $(j, k, l)$.

\section{Conclusions}

In the motion fractal paradigm, a new model on the economic dynamics is established. So:

i) Assimilating any economic system with a complex system, the said system is behaving as a fractal medium. In other words, the economic system becomes a fractal in the most general Mandelbrot sense.

ii) The fundamental assumption of the author's proposed model is the one that the dynamics of any entity of an economic system is defined by continuous but non - differentiable motion curves (fractal motion curves). These fractal motion curves display the property of self - similarity in its every point, which can be converted into a property of holography (every part reflects the whole).

iii) In the previously - mentioned context, the authors discuss about "holographic implementations" of economic dynamics in any economic system through Schrödinger - type fractal "regimes" (i.e. describing economic dynamics through Schrödinger - type equations at various scale resolutions).

iv) Through a special invariance of SL(2R) - type of the Schrödinger type fractal equation, various economic dynamics, in the form of synchronization between any entities of the economic system, are highlighted. More precisely, by means of SL $(2 \mathrm{R})$ - type group, the phase is only moved with a quantity depending on the amplitude of the economic system entity at the transition among various entities of the economic system. More than that, the amplitude of the entity of the economic system is also affected from a homographic perspective. The usual "synchronization" revealed through the delay of the amplitudes and phases of the entities of the economic system must serve here only a fully particular case. 
v) In a particular case of synchronization of economic system entities, given by Riccati type gauge, period doubling, damping oscillations, self modulation and chaotic regimes emerge as natural behaviors in the economic dynamics.

\section{REFERENCES}

Agop M., Mercheș I., Operational Procedures Describing Physical Systems, CRC Press (2018).

Agop M., Păun V.P., On the New Perspectives of Fractal Theory. Fundaments and Applications, Romanian Academy Publishing House, Bucharest (2017).

Arthur W.B., Complexity Economics: A Different Framework for Economic Thought (2013).

Bar-Yam Y., Dynamics of Complex System, Reading, Mass: Perseus Books (1999).

Battiston S., Farmer J.D., Flache A., Garlaschelli D., Haldane A.G., Heesterbeek H., Hommes C., Jaeger C., May R., Scheffer M., Complexity Theory and Financial Regulation. Science, 351(6275), 818-819 (2016).

Cartan E., Riemannian Geometry in an Orthogonal Frame, World Scientific Publishing, Singapore (2001).

Cartan E., La Théorie des Groupes Finis et Continus et la Géométrie Différentielle Traitées par la Méthode du Repère Mobile, Gauthier-Villars, Paris (1951).

Cristescu C.P., Nonlinear Dynamics and Chaos. Theoretical Fundaments and Applications, Romanian Academy Publishing House (2008).

De Alfaro V., Fubini S., Furlan G., Conformal Invariance in Quantum Mechanics, Il Nuovo Cimento A, 34, 569-611, 1976.

Deffner S., Campbell S., Quantum Thermodynamics: An Introduction to the Thermodynamics of Quantum Information, IOP Concise Physics (2019).

Gavriluţ A., Mercheş I., Agop M., Atomicity Through Fractal Measure Theory: Mathematical and Physical Fundamentals with Application, Springer, $1^{\text {st }}$ Edition (2019).

Gemmer J., Michel M., Mahler G., Quantum Thermodynamics, Springer, 2004.

Jackson E.A., Perspectives of Nonlinear Dynamics, 1, Cambridge University Press, New York, 1992.

Mandelbrot B.B., The Fractal Geometry of Nature, W.H. Freeman and Co., San Fracisco (1982).

Mantegna R.N., Stanley H.E., An Introduction to Econophysics: Correlations and Complexity in Finance, Cambridge, UK: Cambridge University Press (2016).

Mazilu N., Agop M., Skyrmions. A Great Finishing Touch to Classical Newtonian Philosophy, New York, Ny Nova Science Publishers C. (2012).

Mazilu N., Agop M., Mercheş I., The Mathematical Principles of Scale Relativity Physics: The Concept of Interpretation, CRC Press (2019).

Mercheş I., Agop M., Differentiability and Fractality in Dynamics of Physical Systems, World Scientific, New Jersey (2016).

Mitchell M., Complexity. A Guided Tour, New York: Oxford, Oxford University Press (2011). 
Niederer P., The Maximal Kinematical Invariance Group of the Free Schrodinger Equation, Helvetica Physica Acta, 45, 802-810, 1972.

Nottale L., Scale Relativity and Fractal Space-Time: A New Approach to Unifying Relativity and Quantum Mechanics, Imperial College Press, London (2011).

Politi A., Badii R., Complexity: Hierarchical Structures and Scaling in Physics Cambridge: Cambridge University Press (2003).

Postnikov M.M., Leçons de géométrie: groupes et algèbres de Lie. Mir, Moscow. (1985).

Simon B., Representations of Finite and Compact Groups, Providence, Ri American Math. Soc. C, 2008.

\section{TIPURI DE DINAMICI HOLOGRAFICE ALE ȘTIINȚEI COMPLEXITĂȚII CU APLICAȚII ÎN DOMENIUL ECONOMIC}

(Rezumat)

Asimilând orice sistem economic complex cu un fractal în cel mai general sens a lui Mandelbrot, sunt analizate comportamente dinamice nediferențiabile ale acestor sisteme. Astfel, dinamici economice sub forma unor diverse regimuri de tip Schrödinger implică ,,implementări holografice” ale proceselor economice prin grupuri invariante de tip SL (2R). Aceste grupuri funcționează ca grupuri de sincronizare între entitătile oricărui sistem economic, situație în care atât fazele cât și amplitudinile acestor entități sunt afectate dintr-o perspectivă homografică. Sincronizarea uzuală manifestă prin întârziere atât în fază cât și în amplitudine a entităților sistemului economic reprezintă aici un caz cu totul particular. Într-un caz special al sincronizării entităţilor sistemelor economice dată prin etalonări de tip Riccati, procesele economice "suportă" scenarii de evoluții spre haos de tip dublare de perioadă, oscilații amortizate, automodulare și regimuri complexe. 